\section{Stanisław Bazyliński OFMConv}

Pontificia Facoltà Teologica San Bonaventura di Roma bazylinski@biblico.it

DOI: http://dx.doi.org/10.12775/BPTh.2017.024
Biblica

et

Patristica

Thoruniensia

10 (2017) 4: 477-491

ISSN (print) 1689-5150

ISSN (online) 2450-7059

\title{
Annotazioni sul testo del Salmo 116
}

\section{Notes on the Text of Psalm 116}

\section{Uwagi o tekście Psalmu 116}

Sommario. Larticolo discute diverse questioni filologiche e testuali che sono fondamentali per una corretta l'analisi del Salmo 116. Dallo studio emergono indizi linguistici che convalidano una datazione postesilica del testo. Inoltre, le attestazioni manoscritte ebraiche depongono a favore della composizione unitaria.

Abstract. This article deals with many philological and textual issues of fundamental weight for an accurate analysis of Psalm 116. The study isolates linguistic evidence that substantiate a post-exilic date of the text. Furthermore, Hebrew manuscripts attest to its unitary composition.

Streszczenie. Artykuł podejmuje wiele zagadnień z filologii i krytyki tekstu, będących podstawą ścisłej analizy Psalmu 116. Studium wyodrębniło elementy językowe, które uzasadniają pochodzenie tekstu z okresu po niewoli babilońskiej. Materiały źródłowe z rękopisów hebrajskich przemawiają za spójnością kompozycji.

Parole chiave: Salmo 116; questioni filologiche; critica del testo; datazione postesilica; composizione unitaria.

Keywords: Psalm 116; philological issues; textual-criticism; post-exilic dating; unitary composition.

Słowa kluczowe: Psalm 116; zagadnienia filologiczne; krytyka tekstu; powygnaniowy czas powstania; jednolita kompozycja.

Tl testo masoretico del Salmo 116 propone al lettore delle problematiche alle quali i commentatori hanno offerto risposte differenti. Si discute sul suo genere letterario, sulla causa che ha ispirato questo canto e sulla sua datazione. Si 
dibatte inoltre se il salmo in questione sia una composizione unitaria o sia composto di due salmi (114 e 115), che sono stati conservati nei Settanta (LXX), nella Vetus Latina (VL) e nella Volgata (Vg). Infine, è al vaglio degli studiosi il ruolo che il Sal 116 svolge nel quinto libro del Salterio e all'interno dei Sal 113-118 ("hallel egiziano"), nonché il suo rapporto con il Sal 115. Non rientra nello scopo di questo contributo rispondere minuziosamente a domande così complesse; quello che ci proponiamo, invece, è di esaminare lo stato del testo e i suoi problemi filologici in modo da preparare il campo a qualsiasi altro tipo di indagine, cercando per di più di isolare indicazioni per una datazione e fornire elementi relativi alla composizione nel suo insieme. Di conseguenza analizzeremo ciascun versetto, soffermandoci sugli aspetti più importanti per la critica testuale e la traduzione.

\section{1 'āhabtî kî-yišma' yhwh|'et-qôlî tahănûnāy}

I LXX, la VL e la Vg premettono al Sal 116,1 il termine $\alpha \lambda \lambda \lambda \eta \lambda$ oviá/alleluia, preso dal Sal 115,18. Il testo masoretico, privo di hal $l \hat{u}-y \bar{a} h$, è confermato dalla Peshitta (Syr), dal salterio iuxta Hebraeos (Hier) e dal Targum (Tg).

Il verbo 'āhabtî all'inizio del v.1 non è seguito da un complemento oggetto secondo il peculiare stile del salmista (cf. 'eqrä', v.2b; he'ěmantî, v.10a), ma da un $k \hat{\imath}$ causale (LXX ötı, VL, Vg, Hier quoniam) ${ }^{1}$. Sebbene 'āhabtî sia reso con

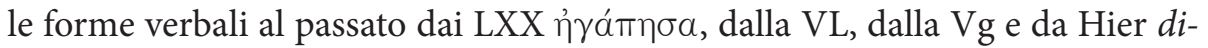
lexi, il qatal di ' $h b$ indica lo stato emotivo e mentale contingente dellorante ed è perciò traducibile con il presente.

Il valore dello hireq in qôli è discusso. Alcuni sospettano la presenza di uno hireq paragogico ${ }^{2}$, poiché nelle altre ricorrenze del sintagma qôl tahănûn (pl.) (Sal 28,2.6; 31,23, 86,6; 130,2; 140,7) cè sempre qôl. Questa ipotesi potrebbe essere corroborata dai LXX (Syr, VL, Vg, Hier) che non traducono il suffisso $-\hat{\imath}^{3}$. Ciononostante si tratta soltanto di un "ragionevole sospetto". I LXX (e le versioni) si comportano, infatti, in modo diverso nella traduzione di una catena appositiva di due nomi comuni muniti entrambi del suffisso della 1a sg. ${ }^{4}$

1 Cf. W. Gesenius, Thesaurus, p. 679. Invece, C.A. \& E.G. Briggs, Psalms, p. 398, attribuisce al kî nei vv.1.2 un valore asseverativo («verly»); da parte sua D. Erbele-Küster, Atempause, p. 214, considera enfatica («Ja») la congiunzione kî dei vv.1.10.

2 P. Joüon, T. Muraoka, A Grammar, \$93p; cf. W. Gesenius, E. Kautzsch, Grammatik, $\$ 901$.

3 Cf. inoltre pel manoscritto 153 di B. Kennicott, Vetus Testamentum, vol. II, p. 410.

4 I LXX non sempre traducono tutti e due i pronomi (cf. 2Sam 19,1; 2Re 4,19; Ger $4,19 ;$ Sal 18,$3 ; 55,14 ; 62,3.7 ; 144,2)$; omettono il primo suffisso pronominale, traducendo la costruzione appositiva con una catena costrutta (Es 4,22) o con altre formulazioni (1Sam 
Preferiamo, perciò, mantenere il valore pronominale di $-\hat{\imath}$. La punteggiatura masoretica con l'accento rebia sopra qôlî avvalora, poi, la possibilità che si tratti di un'apposizione («la mia voce, le mie implorazioni»), come conferma anche il Tg qly $b^{\varsigma} w t y$, «la mia voce, le mie suppliche» ${ }^{5}$. Ciò corrisponde allo stile del salmista, che si diffonde spesso in precisazioni (cf. vv.3bc.8bc.16ab.18ab.19ab).

\section{${ }^{2} k \hat{\imath}$-hittâ 'oznô lî $\mid \hat{u} b^{e} y \bar{a} m a y$ 'eqrä'}

Sebbene nel v.2 la sequenza qatal... yiqtol di hittâ... 'eqrä' sia tradotta con

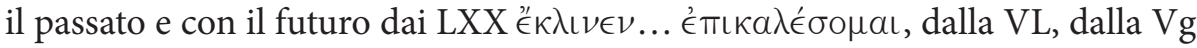
e da Hier inclinavit... invocabo - in modo che ciò che è stato già esaudito (v.2a) preceda ciò che sarà in futuro (v.2b) -, ci sembra possibile rendere lo yiqtol ugualmente con una forma passata. Riteniamo anzitutto corretta la resa dello $\mathrm{x}$-qatal (kî-hitțâ) con l'aoristo o il perfetto, per indicare una circostanza unica nel passato. Va notato, inoltre, che lo x-yiqtol ( $\hat{u} b^{e} y \bar{a} m a y$ 'eqrä') è retto dal kî iniziale ed è collegato con lo x-qatal attraverso il waw copulativo $(\hat{u}-)$. Di conseguenza, lo x-yiqtol appartiene alla stessa sfera passata (cf. 1Re 21,6; Ger 36,18), avendo con ogni probabilità un valore iterativo in virtù all'espressione avverbiale $b^{e} y \bar{a} m a y$. La variante masoretica $\hat{u} b^{e} y \bar{a} m a y$, lett. «e nei miei giorni», trova, poi, appoggio nei LXX, nella VL, nella Vg, in Hier e nel Tg. Invece, la Syr legge il singolare bywm, "nel giorno», facilitando la traduzione per motivi stilistici.

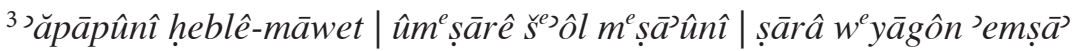

Nel v.3, ventiquattro manoscritti omettono il waw di $\hat{u} m^{e} s \underline{a} r \hat{e}^{6}$ che non è riportato neppure dai LXX, dalla VL e dalla $\mathrm{Vg}^{7}$. La sua presenza va però difesa in ragione della testimonianza della Syr, di Hier e del Tg. Traducendo et munitiones, «e fortificazioni, baluardi», Hier suggerisce come Vorlage la lezione $\hat{u}^{e}{ }^{e} \bar{o} d \hat{e}^{8}$, che è accolta dall'apparato della $B H K$. Tuttavia, è preferibile ûm ${ }^{e} s \bar{a} r \hat{e}$ del testo masoretico con il significato di «e pericoli/strette». Questo senso è fa-

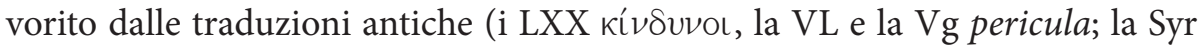

26,17 ; Sal 22,2; 31,11), oppure tralasciano la parola ripetuta (2Sam 19,5), tutti e due i suffissi (2Re 2,12; cf. 13,14) o il secondo suffisso (Is 43,20; Sal 68,25).

5 Cf. B. Walton, Biblia, vol. III, p. 272; D.M. Stec, The Targum, p. 207. Invece L. Díez Merino, Targum, p. 299, traduce «vocem depracationis mee».

6 Cf. B. Kennicott, Vetus Testamentum, vol. II, p. 410.

7 Va precisato che una decina di manoscritti della Vg include la congiunzione et (cf. l'apparato critico dell'edizione dei monaci di San Girolamo).

8 Cf. D.J.A. Clines, The Dictionary, vol. V, p. 445; W. Gesenius, Handwörterbuch, p. 723. Il lessema māṣōd indica anche la trappola per la caccia e pesca (cf. Gb 19,6; Qo 7,26); cf. F. Zorell, Lexicon, p. 465. 
$w^{`} w l s n^{\prime}$, «e oppressioni, tribolazioni», il Tg $w^{`} q t y n$, «e strette») e dai lessicografi moderni ${ }^{9}$.

In coppia con șârâ («oppressione», «angoscia»), il sostantivo yāgôn, «afflizione», mette in risalto la condizione interiore dellorante intrisa di dolore/affli-

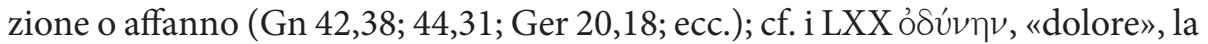
VL, la Vg e Hier dolorem; la Syr $d^{\prime} w w^{\prime} n^{\prime}$, «miseria», «afflizione» ${ }^{10}$; il Tg $d w w n$, «afflizione», «tristezza».

Infine, lo yiqtol 'emșā', con l'aspetto di istantaneità, corrispondente al qatal, «trovai» ${ }^{11}$; cf. i LXX aor. $\epsilon \hat{\mathrm{u}} \rho \mathrm{v}$, la VL e la Vg pf. inveni, Hier pf. repperi.

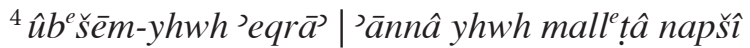

Nel v.4 la preposizione $b^{e}$ in $b^{e} \breve{s} \bar{e} m$ ha probabilmente il valore strumentale di «attraverso», "per mezzo di» ${ }^{12}$ (cf. Gn 4,26; 12,8; 13,4; 21,33; ecc.), benché nelle versioni antiche (LXX, VL, Vg, Hier) e in molte traduzioni moderne la preposizione $b^{e}$ introduca il complemento oggetto; si tratta di rese dettate probabilmente da motivi stilistici delle rispettive traduzioni. Viene così sottolineata la performatività dell'enunciato ${ }^{13}$.

9 L. Alonso Schökel, Diccionario, p. 453: «acoso, asedio del Abismo» («assillo, assedio dell'Abisso»). F. Zorell, Lexicon, p. 467: «angustia, periculum, miseria». E. König, Wörterbuch, p. 241: «Bedrängnis» («assillo», «angustia»). W. Gesenius, Handwörterbuch, p. 726: «die Drangsale d. Unterwelt» («i tormenti degli inferi»). D.J.A. Clines, The Dictionary, vol. V, p. 453: «distress, torment». F. Brown, S.R. Driver, C.A. Briggs, Lexicon, p. 865: «the

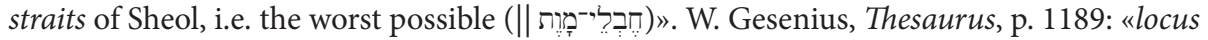
angustus [...] conditio adversa». W. Baumgartner, Lexikon, p. 590, conferisce a mēṣar il significato di «Bedrängnis», anche se per il Sal 116,3 privilegia la congettura $m^{e} s \underline{o} d \hat{e}$.

10 W. Gesenius, Handwörterbuch, p. 435: «Kummer, Gram» («affanno, pena»); F. Zorell, Lexicon, 291: «animi afflictio, tristitia»; W. Gesenius, Thesaurus, p. 563: «moeror, moestitia» («afflizione, tristezza»).

11 P. Joüon, T. Muraoka, A Grammar, \$113h.

12 O. Grether, Name, p. 47: «Da der Name als ein Machtwesen angesehen wurde, konnte er auch als Mittel oder die Vermittlung betrachtet werden, durch die Jahwe wirkt. Deshalb [...] mit ঙ̣, der Präposition des Mittels»; E. Jenni, Die hebräische Präpositionen, vol. I, p. 166: «anrufen unter Verwendung seines Namens»; D. Erbele-Küster, Atempause, p. 216: «"im Namen" oder "mit Namen”».

13 Cf. D. Erbele-Küster, Atempause, p. 216: «In der Rede über das Anrufen wird damit bereits der Gerufene angerufen - während das Ausrufen im Namen Gottes weniger Gott als Gegenüber sieht, als vielmehr Dritte». 
L'interiezione 'ānnâ, «Oh!», «Deh!» (cf. v.16a) $)^{14}$, esprime un desiderio sen-

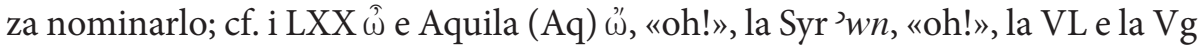
$o$; Simmaco (Sym) $\alpha \hat{\xi} \iota \hat{\omega}$, «consentimi, ti prego».

${ }^{5}$ hannûn yhwh w'ṣaddîq | wè lōhênû me rahềm

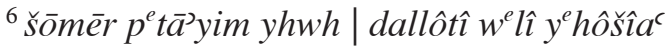

A prima vista, la punteggiatura masoretica di šomēer $p^{e} t \bar{a}^{3} y i m$ (munach sotto $\breve{s} \bar{o} \bar{e} r)$ potrebbe suggerire nel v.6 la presenza di una catena costrutta che denota un attributo di Yhwh («il custode/protettore dei semplici [è] Yhwh»), rafforzando i titoli «benevolo», «giusto» e «compassionevole» del v.5. In realtà, per l'accezione nominale di šômêr si utilizzano altrove altri elementi linguistici $^{15}$, assenti nel v.6. Al contrario, a favore del valore verbale depongono le versioni antiche che introducono un complemento oggetto preceduto dal par-

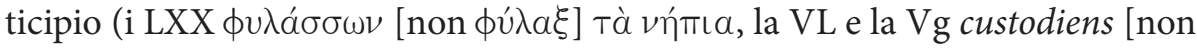
custos] parvulos, la Syr nțr lšbr’, il Tg ntyr šrgygy’) o dal presente indicativo (Hier custodit parvulos). Tale resa corrisponde all'uso frequente di šōmēr con il complemento oggetto senza nota accusativi ${ }^{16}$. Va notato, infine, che a scanso di equivoci il manoscritto 43 inverte l'ordine delle parole (יהוה פתאים (י) (י) $)^{17}$.

Il plurale $p^{e} t \bar{a}^{\prime}$ yim ricorre altre sei volte nella Bibbia (Pro 1,4; 7,7; 8,5; 9,6; $14,18 ; 27,12)$, mentre la forma $p^{e} t \bar{a}{ }^{\prime} i m$, suggerita dall'apparato della $B H K / B H S$, non è mai attestata. Benché se ne discuta l'origine ${ }^{18}$ e l'etimologia ${ }^{19}$, il significato di «inesperti», «semplici» è confermato per il Sal 116,6 anche dalle rese dei LXX

14 Una cinquantina di manoscritti riporta la lezione אנא (cf. B. Kennicott, Vetus Testamentum, vol. II, p. 410), forma presente nel Sal 118,25.

15 Cf. verbi reggenti (1Sam 17,20.22; 28,2; Sal 121,3.4; 127,1), frasi fisse (2Re 22,14 || 2Cr 34,22; Ger 35,4; Est 2,3.8.14.15; Ne 2,8; 3,29), vocativi (Is 21,11.12), parallelismi con altri nomi (Sal 121,5), interrogativi (Gn 4,9).

16 Dt 7,9; $1 \operatorname{Re} 8,23 ; 2 \operatorname{Re} 9,14$; Is 56,2.6; Sal 34,21; 97,10; 146,6; Pro 10,17; 13,3.18; 15,5; 16,$17 ; 19,8.16 ; 21,23 ; 22,5 ; 27,18 ; 29,18$; Qo 8,$5 ; 11,4 ;$ Dn 9,4; Ne 1,5; 9,32; 2 Cr 6,14.

17 B. Kennicott, Vetus Testamentum, vol. II, p. 411.

18 F. Zorell, Lexicon, p. 675 e M. Sæbø, פתה, col. 495, fanno derivare petî da pth, mentre F. Brown, S.R. Driver, C.A. Briggs, Lexicon, p. 834 e W. Gesenius, Handwörterbuch, p. 1089, fanno provenire $p$ th da petî. Infine, W. Baumgartner, Lexikon, p. 925, non sceglie tra queste alternative.

19 Si veda, ad es., W. Gesenius, Handwörterbuch, p. 1089: «ug. PT(Y) verführen [...]; ar. fatā (FTW) u. fatiya jugendlich, jung sein (den. v. fatan Jüngling); äth. fatawa, fatwa begehren, verlangen, tigr. fäta lieben, wünschen». F. Zorell, Lexicon, p. 677 e W. Baumgartner, Lexikon, p. 929, preferiscono l'etimologia araba. M. Sæbø, פתה, col. 495, riassume la discussione (bibliografia). 
$\nu \eta \dot{\pi \iota \alpha}$, della Syr šbr , «bambini» ${ }^{20}$, della VL, dalla Vg e da Hier parvulos; il Tg šrgygy», «semplici, facilmente seducibili».

Il verbo dallôt $\hat{\imath}$, da dll, «essere piccolo», assume il significato metaforico di

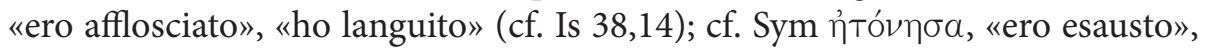
Hier adtenuatus sum, «sono affievolito», piuttosto che quello di «essere umilia-

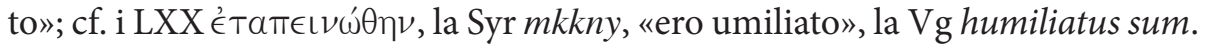
Invece, il Tg rende 'tmsknyt, «sono diventato povero».

La preposizione $l^{e}$ introduce il complemento oggetto (-î) di $y^{e} h \hat{o} s ̌ \hat{\imath} a^{c}$ (cf. Sal 44,4; 72,4; 86,16; 98,1; ecc. ${ }^{21}$; cf. i LXX, Sym, la Vg, Hier). Questa forma del verbo yšc con l'infisso he (cf. 1Sam 17,27), anziché senza (yôšîa $a^{c}$; cf. Sal 34,19; $69,36 ; 72,4.13)$, potrebbe tradire l'influsso dell'aramaico ${ }^{22}$.

${ }^{7}$ šûbî napšs lime ${ }^{e} \hat{u} h \bar{a} y^{e} k \hat{l} \mid k \hat{\imath}-y h w h$ gāmal ' $\bar{a} l \bar{a} y^{e} k \hat{\imath}$

Il termine $m^{e} n u \hat{h} h \bar{a} y^{e} k \hat{\imath}$, lett. «tuoi riposi» (v.7), è un plurale poetico da mānôah, "(luogo / stato di) riposo», "quiete», con il suffisso aramaizzante - $\bar{a} y^{e} k \hat{\imath}$ della 2a sg. f. (cf. Sal 103,3-5) ${ }^{23}$. Il Tg interpreta il nome in senso locale: byt $n w h y k y$, "casa del tuo riposo». Altre versioni (LXX, Syr, Vg, Hier) non chiariscono se si tratti di un luogo o di uno stato. Il salmo suggerisce lopposto della minaccia (v.3), cioè lo stato di riposo, «quiete».

Il verbo gammal, «trattare», «ricompensare» (cf. la Syr $p r^{\wedge} k y$, «ti ha ripagato»; Hier reddet tibi), ha come soggetto Yhwh, il quale dimostra all'orante il proprio favore. Per questo motivo l'espressione gāmal 'a $\bar{a} \bar{a} y{ }^{e} k \hat{\imath}$ si può tradurre «ti ha trattato con bontà», «ti ha fatto del bene», «era buono con te»; cf. i LXX

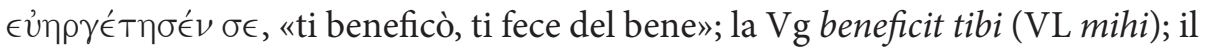
Tg gmyl ț b' 'lyky, «ti ha ricompensato col bene».

${ }^{8}$ kî hillaṣtā napš̂̀ mimmāwet |'et-'ēnî min-dim'â |'et-raglî middeḥ̂̀

Sebbene la forma della 2a sg. m. hillaștā, «hai tratto in salvo, liberato», del testo masoretico (v.8; cf. Sal 56,14), attestata dalla Syr pṣyt e dal Tg pșyt', introduca l'improvviso, enfatico rivolgersi a Yhwh in modo da vivacizzare lo stile (cf. v.4b), essa è preferibile a quella della 3a sg. dei LXX $\epsilon \xi \in$ $\xi \hat{\imath} \lambda \alpha \tau$, della Vg eripuit, della VL e di Hier eruit, tradotta per una maggiore conformità formale con il contesto prossimo.

\footnotetext{
20 B. Walton, Biblia, vol. III, p. 272: «adolescentulos» («giovinetti»).

21 J.P.M. van der Ploeg, Psalmen, vol. II, p. 283, giudica aramaizzante questa costruzi-

22 Cf. P. Joüon, T. Muraoka, A Grammar, $\$ 54 b$.

23 L'uso di questo suffisso aramaico si riscontra nei testi ebraici di Qumran, ad es., in 4Q88 framm. 8,13; 4Q176 framm. 8-11,6.7.12; cf. E. Qimron, The Hebrew, p. 59.
} one. 
Il binomio 'ênî... raglî, «il mio occhio... il mio piede», è al singolare (cf. Tg; invece al plurale nei LXX, nella Syr, nella VL, nella Vg e in Hier) probabilmente in accordo con napšs dallo stesso verbo hillașta.

La frase masoretica min-dim' $\hat{a}$, lett. «dalla lacrima» (collettivo) ${ }^{24}$, ha la grafia priva di nun in 4QPs ${ }^{\circ}$, framm. $2(4 \mathrm{Q} 96)^{25}$ che unisce מדמעה con אתהל] del v.9a. In base allo spazio presente nel frammento qumranico è stata ipotizzata

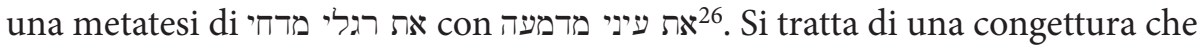
è priva del sostegno delle versioni antiche e di altri manoscritti ebraici.

9 'ethallēk lipnê yhwh | b'arșôt hahayyim

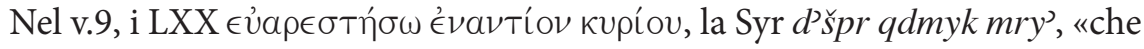
sii grato dinanzi a te, Signore», la VL complacebo ante Dominum, la Vg placebo Domino, suggeriscono apparentemente un verbo diverso da 'ethallēk. Tuttavia, hlk Hitp assume l'accezione di "piacere» in Gn 5,22; 17,1; 24,40; 48,1527; ecc. La traduzione potrebbe essere influenzata dai vv.15-16 che mettono in risalto la lealtà e il legame stretto dell'orante con Yhwh. Questo significato derivato di $h l k$ Hit $^{28}$ non si adatta bene al contesto immediato che, in luogo delle qualità umane, menziona la liberazione operata da Dio (v.8) oppure l'abbandono dell'orante (vv.10-11). Perciò si tratta con ogni probabilità della resa e non di una Vorlage diversa. Infatti, il testo ebraico e il significato di «camminare» sono confermati

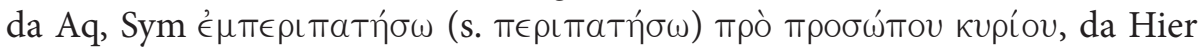
deambulabo coram Domino e dal Tg ’hlk qdm yyy, "camminerò alla presenza di Yhwh».

Il plurale 'arṣôt è attestato da Hier terris, dai manoscritti 116-Z-40 e Héb. 110 del $\mathrm{Tg}^{29}$. Il singolare leggono invece i LXX év $\chi \omega^{\omega} \rho \alpha$, , la Syr $\left.b^{3} r^{(}\right)$, la VL e la $\mathrm{Vg}$ in regione, e due manoscritti ebraici ${ }^{30}$. Il sintagma 'arșôt hahayyîm, essendo peculiare, rappresenta probabilmente una lectio difficilior, dal momento che 'ereș (ha)hayyîm è ricorrente nella Bibbia (cf. Dt 30,19; Is 38,11; 53,8; Ger 11,19; Ez 26,20; 32,23-27.32; Sal 27,13; 52,7; 142,6; Gb 28,13).

24 F. Brown, S. R. Driver, C. A. Briggs, Lexicon, p. 199; W. Baumgartner, Lexikon, p. 218; W. Gesenius, Handwörterbuch, p. 255.

25 Così pure 19 manoscritti di B. Kennicott, Vetus Testamentum, vol. II, p. 411.

26 Così E. Urlich, Qumran, p. 140.

27 J.F. Schleusner, Novus thesaurus, vol. I, p. 927: «Ambulare coram Deo saepe notat Deo placere ob probitatem ac integritatetn vitae».

28 Cf. L. Alonso Schökel, Diccionario, p. 205.

29 Cf. D.M. Stec, The Targum, p. 208.

30 I manoscritti 3, 249 di B. Kennicott, Vetus Testamentum, vol. II, p. 411. 


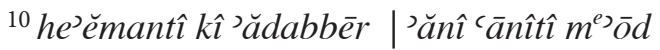

Davanti al v.10, i LXX, la VL e la Vg pongono «alleluia» per segnalare l'inizio di un nuovo salmo. Di questa inserzione non si trovano tracce né nei manoscritti ebraici, né nella Syr, né in Hier, né nel Tg. Inoltre, l’intervallo prima di he'émantî in $4 \mathrm{QPs}^{\circ}$, framm. 2 (4Q96) ${ }^{31}$ confermerebbe questa divisione. Tuttavia, si nota che lo spazio vuoto nel frammento qumranico è frutto di una congettura ispirata dall'influsso dei LXX e della Vg. La cesura dopo il v.9 non è attestata da altri manoscritti ebraici. Invece, alcuni manoscritti fanno iniziare un nuovo salmo nel v.11 (un manoscritto) o nel v.12 (19 manoscritti) ${ }^{32}$.

La Syr rende kî con il waw copulativo, mentre Aq e la Settima lo traducono con ÖTı causale. Invece, per ragioni di logica implicita nellargomentazione e senza tener conto delle versioni antiche, alcuni autori moderni attribuiscono a $k \hat{\imath}$ il valore temporale («quando») ${ }^{33}$ oppure concessivo («anche se», «sebbene» $)^{34}$. Ciononostante queste accezioni paiono meno probabili, considerando che i LXX $\delta \iota^{35}$, la VL, la Vg e Hier propter quod, il Tg 'rwm, «ecco, cosicché», attribuiscono alla congiunzione kî con valore conclusivo di «perciò», "per questo». Esso è attestato in Gn 22,17; Dt 4,22; 2Sam 17,11; Is 7,9; Lam

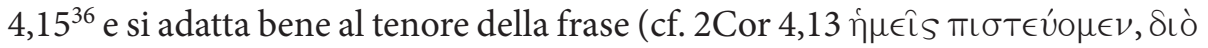
кaì $\lambda a \lambda$ ov̂ $\mu \nu$, «noi crediamo e perciò parliamo»).

31 Cf. E. Urlich, Qumran, pp. 139, 141.

32 B. Kennicott, Vetus Testamentum, vol. II, p. 411, riporta 7 manoscritti, mentre J.B. de Rossi, Variae lectiones, vol. IV, p. 74, cita altri 12.

33 H. Ewald, Die Psalmen, p. 401; W.M.L. de Wette, Commentar, p. 551, citando il Sal 49,19 (LXX ӧтаv, Vg cum); H. Hupfeld, Die Psalmen, vol. IV, p. 234; F. Baethgen, Die Psalmen, p. 349, poiché è la spiegazione più facile («am einfachsten»); G. Castellino, Salmi, p. 422; G. Ravasi, Salmi, vol. III, p. 382, che in nota 5 accetta pure un valore avversativo («benché») e giudica impossibile quello causale (LXX/Vg); L. Alonso Schökel, C. Carniti, Salmos, p. 1412; L.C. Allen, Psalms, p. 151; T. Lorenzin, I Salmi, p. 438, ammettendo in nota 5 la traduzione alternativa: «Io confidavo di poter dire...»; J.-L. Vesco, Le psautier, p. 1090.

34 E.F.C. Rosenmüller, Psalmi, p. 1697; W. Gesenius, Thesaurus, p. 680; C.A. \& E.G. Briggs, Psalms, pp. 398, 399; F. Nötscher, Die Psalmen, p. 236; A. Deissler, Die Psalmen, p. 457; L. Deiss, Je marcherai, p. 39; D.J.A. Clines, The Dictionary, vol. IV, p. 387, apparentemente concessivo («although»); H. Tita, Gelübde, p. 115.

35 Benché $\delta$ ıó in $\mathrm{Tb}$ [S] 14,4 possa assumere una sfumatura causale (dichiarativa in $\mathrm{Tb}$ [S] 3,6), quella conclusiva che esprime la conseguenza dell'enunciato precedente, è usuale.

36 W. Gesenius, Handwörterbuch, p. 73: «so» consecutivo; lo ammette D.J.A. Clines, The Dictionary, vol. IV, p. 387 («therefore»), pur propendendo per il concessivo; cf. A. Agelli, Commentarii, p. 521; G. Heser, Psalmi, p. 919; H. Grotius, Annotationes, vol. I, p. 408; E.W. Hengstenberg, Psalmen, vol. IV, p. 295; B. Duhm, Die Psalmen, pp. 409, 410; H.-J. Kraus, Psalmen, pp. 968, 969; M. Mannati, É. de Solms, Les Psaumes, vol. IV, p. 79; W.A. VanGemeren, Psalms, p. 847; ecc. 
Il senso di «essere umiliato, abbattuto, afflitto, sventurato» per 'ānîtî̀ è con-

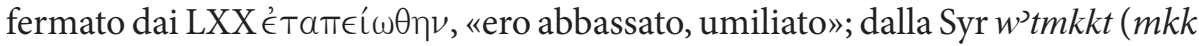
Ethpael), «ed ero umiliato/abbassato»; dalla VL e dalla Vg humiliatus sum, e da Hier adflictus sum.

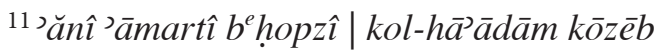

Dal momento che la frase infinitivale $b^{e} h o p z \hat{\imath}$, lett. «nel mio essere costernato» o «quando ero costernato» (v.11) $)^{37}$, trasmette il senso «paura», «angoscia», «costernazione» ${ }^{38}$, si potrebbe renderla anche con «nella mia agitazione» ${ }^{39}$ o «nella mia trepidazione» ${ }^{40}$ (cf. Dt 20,3; Sal 31,23; 48,6; Gb 40,23).

${ }^{12}$ mâ-'āšîb layhwh| kol-tagmûlôhî 'ālāy

Nel v.12, il nome tagmûl è accompagnato dal suffisso aramaico della 3a sg. m. -ôhî (cf. Dn 2,7.18.33.34.46; ecc.) ${ }^{41}$.

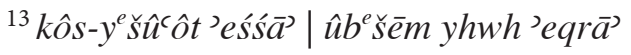

Il plurale $y^{e} \breve{s} \hat{u}^{\top} \hat{o} t$, lett. «salvezze», «liberazioni» (v.13), è tradotto di rego-

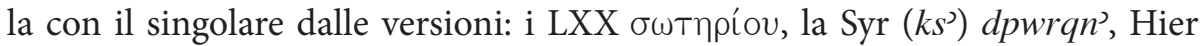
salutis, il Tg $\left(k s^{\prime}\right) d p w r q n^{\prime}$. Anche se Sym è frammentario, la sua resa u̇tè $\rho$

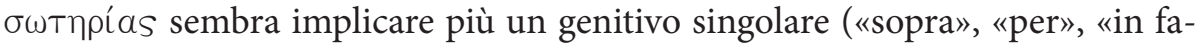
vore di») che l'accusativo plurale («sopra», «oltre», «prima di»).

Invece, l'aggettivo salutaris, «(calice) salutare/benefico», della VL e della Vg non consente una scelta univoca. I vocabolari preferiscono prudentemente il singolare $^{42}$ al plurale ${ }^{43}$. Dal punto di vista grammaticale, la resa di $y^{e} \breve{s} \hat{u}^{c} \hat{o} t$ al singolare può essere spiegata come un "plurale di potenziamento", fenomeno attestato spesso per il plurale dei nomi astratti ${ }^{44}$ ed anche per $y^{e} \breve{s} \hat{u}^{c} \hat{o} t$ (cf. Sal 28,$8 ; 42,6.12 ; 43,5$ ).

37 F. Zorell, Lexicon, p. 258: «consternatus est».

38 W. Gesenius, Handwörterbuch, p. 379.

39 W. Gesenius, Thesaurus, p. 505: «in pavore meo».

40 G. Castellino, Salmi, p. 106.

41 Cf. P. Joüon, T. Muraoka, A Grammar, $\$ 94$ i. Questo suffisso si riscontra nei testi ebraici di Qumran, ad es., in 1QS 5,3.11; 6,13.26 (1Q28); cf. E. Qimron, The Hebrew, p. 61; G. A. Rendsburg, Qumran Hebrew, p. 242.

42 W. Gesenius, Thesaurus, p. 695; F. Zorell, Lexicon, p. 336; E. König, Wörterbuch, p. 165; W. Baumgartner, Lexikon, p. 426; L. Alonso Schökel, Diccionario, p. 342; D.J.A. Clines, The Dictionary, vol. IV, p. 377; W. Gesenius, Handwörterbuch, p. 507.

43 F. Brown, S. R. Driver, C. A. Briggs, Lexicon, p. 447; D.J.A. Clines, The Dictionary, vol. IV, p. 331.

44 Cf. E. König, Historisch-comparative Syntax, \$262e (p. 203); E. König, Wörterbuch, p. 165; C. Brockelmann, Hebräische Syntax, \$19b. Sei manoscritti ebraici leggono il singo- 
${ }^{14} n^{e}$ dāray layhwh 'ăšallēm | neg ${ }^{e}$ dâ-nnā' l'kol-‘ammô

Sebbene il v.14 manchi nei LXX e nella VL, esso è presente nei manoscritti ebraici ed è attestato da una parte delle testimonianze greche (cf. apparato dell'edizione di Göttingen) ${ }^{45}$ e da altre traduzioni antiche (Syr, Vg, Hier, Tg).

La costruzione $n e g^{e} d \hat{a}-n n \bar{a} l^{e}$, «davanti», «al cospetto di» (cf. v.18b, seguito nel v.19 da due frasi che fanno riferimento al luogo: «negli atri», «in mezzo a te»), corrisponde essenzialmente a $l^{e}$ neged (cf. 2 Re 1,13). Il suffisso - $\hat{a}$, atono, è verosimilmente un'aggiunta poetica che non cambia il significato di neged ${ }^{46}$. La particella $n \bar{a}$, ommessa nelle traduzioni, ha un valore enfatico di "pure», «sì (cf. Sal 115,2).

\section{${ }^{15} y \bar{a} q \bar{a} r b^{e} \hat{e} n e \hat{e} y h w h \mid$ hammāaw $w^{e}$ â lahăsî̀āyw}

La resa letterale di $b^{e} \hat{e} n \hat{e}$ yhwh, «agli occhi di Yhwh» (v.15), è evitata per motivi teologici dai LXX évavTíov kupíov (Sal 72[71],14; cf. Is 43,2) ${ }^{47}$, dalla VL, dalla Vg e da Hier in conspectu Domini, che si preoccupano dello stile.

ישועת (cf. B. Kennicott, Vetus Testamentum, vol. II, p. 411, manoscritti 4, 37, 131, 176, 249, 268), forse per evitare la difficoltà interpretativa del plurale.

45 Questo versetto è, invece, inserito in corpore dall'edizione di R. Holmes; J. Parsons, Vetus Testamentum, vol. III, Oxonii 1823, ad locum e tra i Padri greci, lo riportano Giovanni Crisostomo, Exp. in Ps., Patrologia Graeca 55, col. 325 e Teodoreto di Ciro, Int. in Ps., Patrologia Graeca 80, col. 1804B.

46 Cf. W. Gesenius, E. Kautzsch, Grammatik, $\$ 90 f ;$ P. Joüon, T. Muraoka, A Grammar, $\$ 93 \mathrm{c} ;$ H. Bauer, P. Leander, Historische Grammatik, $\$ 72 \mathrm{~g}$ («in Gegenwart des»); R. Meyer, Grammatik, vol. II, $\$ 52.1 \mathrm{~d}$ («in Gegenwart von»). Pur riconoscendo il carattere poetico di $n e g^{e} d \hat{a}-n n \bar{a}$, H. Ewald, Lehrbuch, $\$ 246$, pensa che la costruzione esprima un desiderio di fare qualcosa («o doch vor... [will ich es thun]»). J.P. Fokkelman, G.A. Rendsburg, נגדה נא לכל עמו , pp. 328-336, ritengono, invece, ngdh come imperativo lungo Qal da $n g d$, "guidare», «condurre» (nig ${ }^{e}$ dâ?) (p. 329) e offrono due traduzioni alternative: «lead now, for the benefit of all his people» e «lead now his entire people» (p. 336). Benché questa proposta voglia essere fedele agli «ancient tradents» (p. 328, n. 2) e risolvere, così, la difficoltà dell'unicità (e dell'anormalità in cerca di soluzione) della costruzione (una preposizione seguita dalla particella $n \bar{a}$, p. 328; la costruzione è attestata in Es 12,9: mimmennû $n \bar{a}$ ), essa si scontra proprio con quelle traduzioni antiche che considerano $n g d h$ una preposizione: i LXX Évavtíov, la Syr $q d m$, la Vg e Hier in conspectu. Inoltre, l'assenza della particella $n \bar{a}^{3}$ nel v.14 in un manoscritto di B. Kennicott, Vetus Testamentum, vol. II, p. 411 e nel v.18 in 5 manoscritti di Kennicott e in 4 manoscritti di J.B. de Rossi, Variae lectiones, vol. IV, p. 75, attesta il tentativo di mantenere il valore preposizionale di $n g d h$. Si veda inoltre Rashi

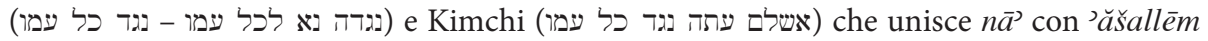
(נא דבק עם אשלם, לא עם נגדה), interpretando il v.14 non come una dichiarazione, ma una richiesta: «Che io possa adempiere i miei voti davanti a tutto il suo popolo».

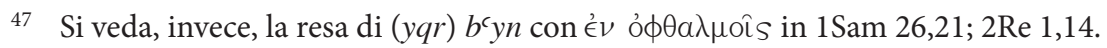


Come nel v.14, il suffisso - $\hat{a}$, atono, è verosimilmente un'aggiunta poetica che non cambia il significato della parola hammā $w^{e} t \hat{a}^{48}$. Quanto poi alla preposizione $l^{e}$, che introduce il complemento di specificazione (laḥ̆âîāayw), essa è di uso tardivo ${ }^{49}$.

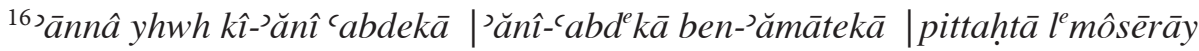

Anche se la congiunzione kî (v.16), manca nei LXX e nella Syr, essa va conservata seguendo la Vg, Hier e il Tg. La preposizione $l^{l}$ davanti a môsērāay introduce, poi, il complemento diretto che, secondo i grammatici ${ }^{50}$, è tipico del periodo tardo. Infine, il sostantivo môsêr significa «catene», «legami».

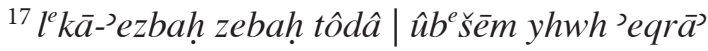

Nel v.17, il sintagma zebah tôdâ è reso dalle versioni in modi diversi: i LXX

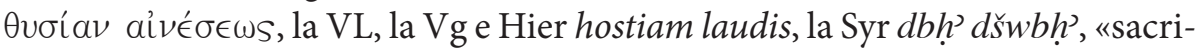
fici di lode»; il Tg $d b h n_{k s t}$, «sacrificio d'immolazione» ${ }^{51}$. Nei lessici moderni, tôdâ è considerata da diversi punti di vista: cè chi propone il significato concreto di «sacrificio di ringraziamento» («Dankopfer») e chi si chiede se attraverso il significato di «canto di ringraziamento» («Danklied») sia diventato la

48 Cf. W. Gesenius, E. Kautzsch, Grammatik, $\$ 90 f ;$ H. Bauer, P. Leander, Historische Grammatik, \$65t; P. Joüon, T. Muraoka, A Grammar, \$ 93i. W. Gesenius, Thesaurus, p. 780: "i parag[ogicum].», usato in maniera pleonastica senza un significato proprio. Invece, R. Meyer, Grammatik, vol. II, $\$ 52.3 \mathrm{a}$, attribuisce a he un valore di avverbio locale («zum Tode hin»); cf. D.J.A. Clines, The Dictionary, vol. V, p. 199: «ה of direction»; cf. E. König, Die Psalmen, p. 448: "Gar schwer ist in den Augen Yahwes die Preisgabe seiner Frommen in den Tod»; cf. idem, Wörterbuch, p. 215; J.A. Emerton, How, p. 147, opta per he locale pleonastico. A giudizio di P.C.H. Wernberg-Møller, The Old Accusative, p. 156, la finale - $\hat{a}$ rappresenta l'accusativo («the use of the accusative instead of the nominative as indicating the semantic subject is in accordance with comparable syntactical structures to be found elsewhere in the Hebrew Bible»). Questa scelta riprende la proposta di C. Siegfried, B. Stade, Wörterbuch, p. 334 e quella di M. Dahood, Psalms III, p. 150 (cf. H. Hupfeld, Die Psalmen, vol. IV, pp. 229-230; F. Baethgen, Die Psalmen, p. 348; A.B. Ehrlich, Die Psalmen, p. 294). Lo studioso danese ritiene (p. 164), inoltre, che hammāw $w^{e} \hat{a}$, forma lunga arcaica, sarebbe stata utilizzata per motivi metrici (come anche negdâ nei vv.14.18), fornendo al poeta una sillaba supplementare: in caso contrario il v.15 potrebbe risultare troppo corto in confronto con il v.15a.

49 Cf. W. Gesenius, E. Kautzsch, Grammatik, \$129.

50 Cf. ibidem, $₫ 117 \mathrm{n}$.

51 Probabilmente in virtù del contesto del salmo i vocabolari propendono per il sacrificio di ringraziamento; cf. L. Alonso Schökel, Diccionario, pp. 793-794; W. Gesenius, Handwörterbuch, p. 1427; D.J.A. Clines, The Dictionary, vol. VIII, p. 598. Si veda, inoltre, la resa

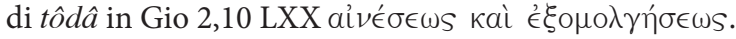


parola per «ringraziamento» («Dank») - accezione possibile per i Sal 50,14.24; $116,17^{52}$; o ancora se tôdâ sia un genitivo epesegetico ${ }^{53}$.

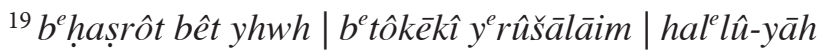

In $b^{e} t \hat{o} k \bar{e} k \hat{\imath}$ (v.19), il suffisso della 2a sg. f. -ēkî è una forma aramaizzante ${ }^{54}$; si veda inoltre nel v.7 il suffisso della $2 \mathrm{a}$ sg. f. $-\bar{a} y^{e} k \hat{\imath}$ in $\lim ^{e} n u \hat{h} h \bar{a} y^{e} k \hat{\imath},{ }^{\prime} \bar{a} l \bar{a} y^{e} k \hat{l}^{55}$.

L'hal $l \hat{u}-y \bar{a} h$ finale è omesso dalla Syr; invece, i LXX, la VL e la Vg lo premettono al versetto seguente. Il Tg segue il testo masoretico.

\section{Conclusione}

Dalle indagini svolte emergono alcuni indizi linguistici in favore di una datazione tardiva (postesilica) del salmo, senza tuttavia determinare se si tratti della seriorità originaria o redazionale. Questi indizi includono gli aramaismi come, ad esempio, i tre suffissi della 2a sg. f. (vv.7bis.19) e uno della 3a sg. m. (v.12), e l'uso della preposizione $l^{e}$ al posto del complemento oggetto (vv.6.16); a ciò si aggiungono le forme con he paragogico (vv.14.15).

La tradizione manoscritta ebraica, inoltre, favorisce l'inclusione del v.14 (cf. Syr, Vg, Hier, Tg), eliminato dai LXX e dalla VL, e non sostiene la divisione dei LXX, della VL e della Vg in due salmi. Infatti, prima del v. 10 è assente $h^{\prime} l \hat{u}-y \bar{a} h$, che le suddette versioni mettono all'inizio del Sal 115 (116,10-19 del testo masoretico). Anche se un manoscritto ebraico (fine del XIII sec.) fa iniziare un nuovo salmo nel v.11 e una ventina di manoscritti (tra il XIII e il XV sec.) nel v.12, queste divisioni non trovano fondamento in nessuna traduzione antica e nella maggioranza dei manoscritti ebraici. In breve, la documentazione manoscritta depone sostanzialmente a favore di un testo unitario più che di due salmi distinti.

\section{Bibliografia}

Agelli A., Commentarii in psalmos et in divini officii cantica, Romae 1606.

Allen L.C., Psalms 101-50 (Revised), Word Biblical Commentary 21, Nashville 2002.

\footnotetext{
52 W. Baumgartner, Lexikon, pp. 1562-1563.

53 F. Zorell, Lexicon, p. 890.

54 Cf. W. Gesenius, E. Kautzsch, Grammatik, §91e; P. Joüon, T. Muraoka, A Grammar, $\S 94 \mathrm{~h}$.

55 P. Joüon, T. Muraoka, A Grammar, $\$ 94 \mathrm{i}$, considera questo suffisso una forma aramaizzante del dialetto settentrionale.
} 
Alonso Schökel L., Diccionario bíblico hebreo-español, Madrid 1994.

Alonso Schökel L., Carniti C., Salmos. Traducción, introducciones y comentario (Salmos 73-150), Estella 1993.

Baethgen F., Die Psalmen, Handkommentar zum Alten Testament 2/2, Göttingen ${ }^{3} 1904$.

Bauer H., Leander P., Historische Grammatik der hebräischen Sprache des Alten Testamentes, Halle a.S. 1922.

Baumgartner W. et al., Hebräisches und aramäisches Lexikon zum Alten Testament, vol. I-V, Leiden ${ }^{3} 1967-1996$.

Briggs C.A. \& E.G., A Critical and Exegetical Commentary in the Book of Psalms, vol. II, International Critical Commentary, Edinburgh 1907.

Brockelmann C., Hebräische Syntax, Neukirchen 1956.

Brown F., Driver S.R., Briggs C.A., A Hebrew and English Lexicon of the Old Testament, Oxford 1906.

Castellino G., Libro dei Salmi, Sacra Bibbia, Torino-Roma 1955.

Clines D.J.A., The Dictionary of Classical Hebrew, vol. IV, Sheffield 1998.

Clines D.J.A., The Dictionary of Classical Hebrew, vol. V, Sheffield 2001.

Clines D.J.A., The Dictionary of Classical Hebrew, vol. VIII, Sheffield 2011.

Dahood M., Psalms III, Anchor Bible 17A, Garden City 1970.

de Rossi J.B., Variae lectiones Veteris Testamenti, vol. IV, Parmae 1788.

Deiss L., Je marcherai en présence de Yahweh (Psaume 116; Vulgate: 114-115), Bible et vie chrétienne 39 (1961), pp. 37-53.

Deissler A., Die Psalmen, Die Welt der Bibel, Düsseldorf 1977.

Díez Merino L. (ed.), Targum de Salmos. Edición Príncipe del Ms. Villa-Amil n. 5 de Alfonso de Zamora, Bibliotheca Hispana Bíblica 6, Madrid 1982.

Duhm B., Die Psalmen, Kurzer Hand-Commentar zum Alten Testament 14, Tübingen ${ }^{2} 1922$.

Ehrlich A.B., Die Psalmen, Berlin 1905.

Emerton J.A., How Does the Lord Regard the Death of His Saints in Psalm cxvi. 15?, Journal of Theological Studies 34 (1983), pp. 146-156.

Erbele-Küster D., Atempause: Eine kleine Poetik des Gottesnamens ausgehend von Ps 116, in Gottes Name(n). Zum Gedenken an Erich Zenger, ed. I. Müllner, L. Schwienhorst-Schönberger, R. Scoralick, Herders biblische Studien 71, Freiburg i.B.-Basel-Wien 2012, pp. 211-226.

Ewald H., Ausführliches Lehrbuch der hebräischen Sprache des Alten Bundes, Leipzig ${ }^{6} 1855$.

Ewald H., Die Psalmen und die Klagelieder, Die Dichter des Alten Bundes 1/2, Göttingen ${ }^{3} 1866$.

Fokkelman J.P., Rendsburg G.A., ngdh n' lkl 'mw (Psalm cxvi 14B, 18B), Vetus Testamentum 53 (2003), pp. 328-336.

Gesenius W., Thesaurus philologicus criticus linguae hebraeae et chaldaeae Veteris Testamenti, vol. I-III, Lipsiae ${ }^{2} 1829-1853$.

Gesenius W., Hebräisches und aramäisches Handwörterbuch über das Alte Testament, vol. I-VI, Berlin-Heidelberg ${ }^{18} 1987-2010$.

Gesenius W., Kautzsch E., Hebräische Grammatik, Leipzig ${ }^{28} 1909$. 
Giovanni Crisostomo, Expositio in Psalmos, ed. J.-P. Migne, Patrologia Graeca 55, Parisiis 1862, coll. 35-498.

Grether O., Name und Wort Gottes im Alten Testament, Beihefte zur Zeitschrift für die alttestamentliche Wissenschaft 64, Gießen 1934.

Grotius H., Annotationes in Vetus Testamentum, vol. I, Halae 1775.

Hengstenberg E.W., Commentar über die Psalmen, vol. IV, Berlin 1845.

Heser G., Psalmi Davidis regis centum et quinquaginta. Juxta sensum literale explanati, Ingolstadij 1678.

Holmes R., Parsons J., Vetus Testamentum Graecum cum variis lectionibus, vol. III, Oxonii 1823.

Hupfeld H., Die Psalmen, vol. IV, Gotha 1862.

Jenni E., Die hebräische Präpositionen. Die Präposition Beth, vol. I, Stuttgart-BerlinKöln 1992.

Joüon P., Muraoka T., A Grammar of Biblical Hebrew (Revised Edition), Subsidia biblica 27, Roma 2006.

Kennicott B., Vetus Testamentum Hebraicum cum variis lectionibus, vol. II, Oxonii 1780.

König E., Historisch-comparative Syntax der hebräischen Sprache. Schlußteil des historisch-kritischen Lehrgebäudes des Hebräischen, Leipzig 1897.

König E., Die Psalmen, Gütersloh 1927.

König E., Hebräisches und aramäisches Wörterbuch zum Alten Testament, Leipzig 1931.

Kraus H.-J., Psalmen, Biblischer Kommentar Altes Testament 15/2, Neukirchen-Vluyn ${ }^{6} 1989$.

Lorenzin T., I Salmi. Nuova versione, introduzione e commento, I Libri Biblici. Primo Testamento 14, Milano 2000.

Mannati M., de Solms É., Les Psaumes, vol. IV, Cahiers de la Pierre-qui-Vire, Paris 1968.

Meyer R., Hebräische Grammatik, vol. II, Sammlung Göschen 764/764a/764b, Berlin ${ }^{3} 1969$.

Nötscher F., Die Psalmen, Echter Bibel, Würzburg 1947.

Qimron E., The Hebrew of the Dead Sea Scrolls, Harvard Semitic Studies 29, Atlanta 1986.

van der Ploeg J.P.M., Psalmen, vol. II, Boeken van het Oude Testament 7b, Roermond 1974.

Ravasi G., Il libro dei Salmi. Commento e attualizzazione (101-150), vol. III, Collana Lettura Pastorale della Bibbia 17, Bologna 1985.

Rendsburg G. A., Qumran Hebrew (with a Trial Cut [1QS]), in The Dead Sea Scrolls at 60. Scholarly Contributions of New York University Faculty and Alumni, ed. L. H. Schiffman, S. Tzoref, Studies on the Texts of the Desert of Judah 89, Leiden-Boston 2010, pp. 217-247.

Rosenmüller E.F.C., Psalmi annotatione perpetua illustrati, Scholia in Vetus Testamentum 4/3, Lipsiae ${ }^{2} 1823$.

Sæbø M., pth verleitbar sein, in Theologisches Handwörterbuch zum Alten Testament, ed. E. Jenni, C. Westermann, vol. II, München-Zürich 1984, coll. 495-498. 
Schleusner J.F., Novus thesaurus philologico-criticus: sive, lexicon in LXX et reliquos interpretes Graecos, ac scriptores apocryphos Veteris Testamenti, vol. I-III, Londini ${ }^{2} 1829$.

Siegfried C., Stade B., Hebräisches Wörterbuch zum Alten Testamente, Leipzig 1893.

Stec D.M. (ed.), The Targum of Psalms, The Aramaic Bible 16, London-New York 2004.

Teodoreto di Ciro, Interpretatio in Psalmos, ed. J.-P. Migne, Patrologia Graeca 80, Parisiis 1860 , coll. 857-2002.

Tita H., Gelübde als Bekenntnis. Eine Studie zu den Gelübden im Alten Testament, Orbis biblicus et orientalis 181, Freiburg, Schweiz-Göttingen 2001.

Ulrich E. et al. (ed.), Qumran Cave 4. XI. Psalms to Chronicles, Discoveries in the Judaean Desert 16, Oxford 2000.

VanGemeren W.A., Psalms (Revised Edition), Expositor's Bible Commentary 5, Grand Rapids 2008.

Vesco J.-L., Le psautier de David, Lectio divina 210, Paris 2006.

Walton B., Biblia Sacra Polyglotta, vol. III, Londini 1656.

Wernberg-Møller P.C.H., The Old Accusative Case Ending in Biblical Hebrew. Observations on himwth in Ps. 116:15, Journal of Semitic Studies 33 (1988), pp. 155-164.

de Wette W.M.L., Commentar über die Psalmen, Heidelberg ${ }^{5} 1856$.

Zorell F., Lexicon Hebraicum et Aramaicum Veteris Testamenti, Romae 1984. 